\title{
Multifractal Analysis and Compressive Strength Prediction for Concrete through Acoustic Emission Parameters
}

\author{
Zhiqiang $\mathrm{Lv} \mathbb{D},{ }^{1}$ Annan Jiang $\mathbb{D}^{,},{ }^{1}$ Jiaxu Jin, ${ }^{2}$ and Xiangfeng $\mathrm{Lv}^{3}$ \\ ${ }^{1}$ School of Transportation Engineering, Dalian Maritime University, Dalian, Liaoning 116026, China \\ ${ }^{2}$ School of Civil Engineering, Liaoning Technical University, Fuxin, Liaoning 123000, China \\ ${ }^{3}$ School of Civil and Resource Engineering, University of Science and Technology Beijing, Beijing 100083, China \\ Correspondence should be addressed to Annan Jiang; jiangannan@163.com
}

Received 30 November 2020; Accepted 1 February 2021; Published 12 February 2021

Academic Editor: Wei Liu

Copyright ( $\odot 2021$ Zhiqiang Lv et al. This is an open access article distributed under the Creative Commons Attribution License, which permits unrestricted use, distribution, and reproduction in any medium, provided the original work is properly cited.

\begin{abstract}
Acoustic emission (AE) can be applied to identify crack propagation and damage of materials and structures. However, few studies investigate the multifractal regularity and compressive strength prediction for concrete using AE parameters. Therefore, the major objective of this research is to perform multifractal analysis of damage and develop support vector machine (SVM) for strength prediction based on AE parameters. Meanwhile, fuzzy c-means (FCM) was implemented to identify damage mechanisms. The results showed that the level of damage can be revealed qualitatively and quantitatively by analyzing morphology and parameters of multifractal. In particular, the multifractal parameter $\alpha_{0}$ has the ability to identify critical damage and primary failure surface. Moreover, damage mechanisms were further distinguished by FCM. Finally, the results showed that the parameters of AE can further expand the application of AE for predicting compressive of concrete. SVM prediction results using AE parameters perform higher precision than the artificial neural network (ANN). Furthermore, a significant reduction in sample size uses AE parameters to predict concrete strength.
\end{abstract}

\section{Introduction}

Concrete, as one of the most widely used groups of the construction material, is frequently applied to structure because of the favorable properties [1]. However, due to exposure to severe conditions, the existence of cracks has further expanded, resulting in degradation of the physical and mechanical properties [2]. Moreover, there is almost no plastic deformation and significant reduction in structural integrity, when the stress is kept below the proportional limit or yield point. Once the stress of concrete exceeds the yield strength, the development of instability crack causes brittle failure. Therefore, in order to ascertain the safety of structures in service, the reliable monitoring techniques are at the heart of our understanding of evaluating the structural integrity of structure [3]. Acoustic emission (AE) is a potential technique to determine the behavior of concrete according to the signal released by the crack. Recent developments in $\mathrm{AE}$ have heightened the need for the integrity evaluation of material and construction in service with the advantages of high sensitivity and accurate evaluation [4].

$\mathrm{AE}$ is a nondestructive monitoring method with the functions of identifying crack growth and failure mechanism of materials and structures. The signals, collected by the sensor attached to the material, are recorded and processed by the $\mathrm{AE}$ system. A typical $\mathrm{AE}$ signal of Figure 1 is a complex, damped, sinusoidal voltage vs. time plot [5]. The $\mathrm{AE}$ waveform originates from the elastic wave released by the crack containing crack scale, damage mechanism, and damage status $[6,7]$. Several criterions and research studies have been developed for evaluating the damage of concrete based on the different waveform parameters (counts, hits, energy, signal amplitude, and frequency) [8-10]. Kocáb has investigated the characteristics of $\mathrm{AE}$ events corresponding to the crack formation and propagation of cement. The study contests the claim that the qualitative relationship was revealed between the events and cracks of concrete [11]. Aggelis acquired waveform parameters, closely following the 


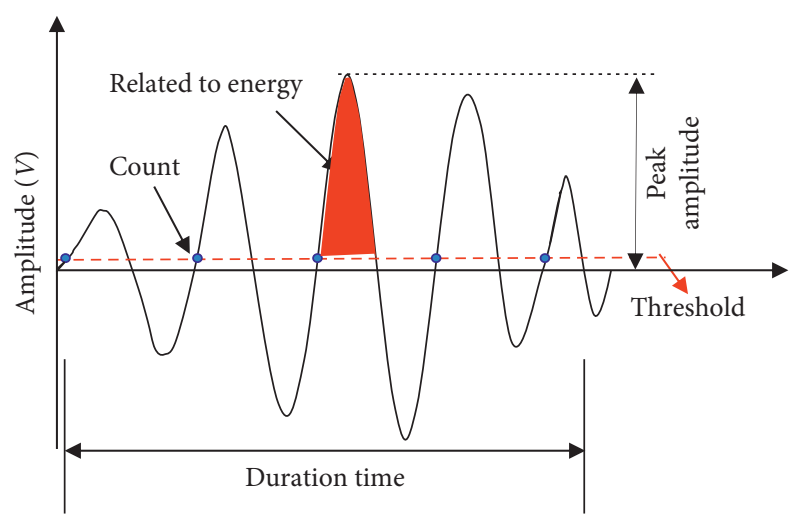

Figure 1: Parameters of typical AE signal [5].

initial microcracking to macrocracking of concrete. It is reasonable to suggest that the waveform parameters can be used to distinguish the different fracturing stages [12]. For most cases, it can be observed that tensile cracks occur in microdamages, resulting in short rise time and high frequency of AE waveforms, followed by shear and multiple shear mixed mode cracks developed in macrodamages, resulting in long rise time and lower frequency of $\mathrm{AE}$ waveforms. Thus, RA and average frequency can exhibit the dominant fracture mode and then show the level of damage of the materials [13]. Researchers also found that statistical analysis can further quantitatively judge on the damage of material [14]. Carpinteri found that AE amplitude is connected with the scale of fracture, following the empirical Gutenberg-Richter's law [15]. The statistical characteristics of $\mathrm{AE}$ amplitude can provide quantitative measurements of damage in the whole failure process [3]. In addition, Abdelrahman proposed a modifying index of damage to identify the yielding point of the structure [4]. Obviously, the statistical analysis of the AE parameters has been brought to public attention. Monofractal and multifractal structures can describe the irregularity of the vibration signal as a whole and reflect the local characteristics of vibration signal more precisely [16]. Furthermore, the variation of dynamic nonlinear multifractal parameters has been used to quantitatively describe complex systems, especially in climate change, earthquake warning, and rock mass instability $[17,18]$. However, few writers have been able to draw on any systematic research into the multifractal dynamic identification of damaged concrete using AE.

It is of profound significance to prove whether the load has reached the crack damage. Therefore, the only way to avoid the abrupt failure of this structure is to reduce the load on the structure. There are various modeling approaches for prediction of concrete (CS) [19]. Artificial intelligence techniques have strong nonlinear capability, good robustness, and generalization ability by establishing a mathematical model to predict concrete strength [20]. However, among all artificial intelligence approaches, the artificial neural network (ANN) and support vector machine (SVM), as two popular and effective methods, have been proved to be successful in scientific research and practical application. SVM has a better performance in processing on a limited number of samples compared with traditional statistical theory. Moreover, SVM is a powerful tool to improve generalization performance by implementing that SRM aims at minimizing a bound on the generalization error of a model rather than minimizing the error on the training data only [21]. The algorithm can be transformed into a convex optimization problem, which can guarantee the global optimality of the algorithm and avoid the local minima which cannot be solved by the neural network. Hence, SVM can achieve robust approximation ability and generalization ability. Several attempts have investigated the possibility of applying SVM in concrete. Lee has been successful in using SVM and ANN to determine concrete strength based on the mix proportion data, and it was observed that SVM estimated the CS of concrete with high prediction precision [22]. Shih predicted that concrete strength combined nondestructive tests and destructive tests. It was concluded that CS of concrete can be accurately estimated based on SVM using nondestructive parameters [23]. Nazari and Abd verified that SVM is a potential tool for prediction of concrete strength by adopting mixture components as input vectors $[24,25]$. However, a host of specimens are required for the above studies. Sasikumar et al. attempted to use AE to predict the strength of 18 fiber materials [26]. As far as known, there are rarely reports on the application of a small number of specimens used to predict the strength of concrete by using AE parameters as input vectors of SVM.

There are two primary aims of this study: (1) to establish the relationship between multifractal statistical analysis of AE parameters and the damage evolution of concrete and (2) to develop a SVM model to estimate the CS of concrete based on $\mathrm{AE}$ parameters. In this research, the AE activity and signatures of concrete were recorded in the whole process of concrete compression with AE monitoring. At first, the damage evolution of concrete is briefly analyzed based on AE parameters and statistical analysis of $b$. Then, the selfsimilarity of the AE signal is explored using multifractal, which provides quantitative and qualitative analyses to the damage evolution. Meanwhile, damage mechanisms were further determined with the help of fuzzy c-means (FCM) in the deformation and fracture of concrete. In the end, the SVM model was developed to estimate the CS of concrete using AE parameters.

\section{Experimental Study}

2.1. Material and Samples Preparation. To obtain AE data of concrete under compression, this experimental study was carried out using concrete specimens with water-cement ratio of 0.45 . The total amount of concrete is 15 in size $100 \mathrm{~mm} \times 100 \mathrm{~mm} \times 100 \mathrm{~mm}$ (two specimens did not meet the requirements). Ordinary Portland cement type 42.5 was used as the binding material. Gravel was adopted as the coarse aggregate $(<25 \mathrm{~mm})$, and dry river sand was adopted as the fine aggregate. Details of all mix proportions of concrete mixtures are listed in Table 1 . The specimens were placed into water saturated with calcium hydroxide at $20 \pm 2^{\circ} \mathrm{C}$ for 28 days until testing. 
TABle 1: Concrete mixture design.

\begin{tabular}{ccccc}
\hline W/b & Water $(\mathrm{kg})$ & Cement $(\mathrm{kg})$ & Sand $(\mathrm{kg})$ & Aggregate $(\mathrm{kg})$ \\
\hline 0.45 & 185 & 411 & 685 & 1118
\end{tabular}

2.2. Compression Test. Compression tests of the concrete specimens were carried out according to JTG E30-2005 standard [27]. The CS of the specimens was tested at a constant loading rate of $3 \mathrm{kN} / \mathrm{s}$. At the same time, AE parameters by the specimens were recorded.

2.3. AE Monitoring of Concrete Compression Process. The DS5 AE testing system, including sixteen channels, was used to monitoring of the concrete compression process. RS-2 A sensors, with the frequency response range from 60 to $400 \mathrm{kHz}$, were used here. The RS-2A sensor is proved to be effective in monitoring by $\mathrm{Ma}$ [28]. Vaseline was used as the coupling agent between the sensors and the concrete surface. Then, the AE sensor was fixed at the center of both sides of the specimen. The environmental noise can be successfully eliminated by the setting of the AE threshold. In this study, AE signal threshold is $180 \mathrm{mV}$. The gain of preamplifier and main amplifier is $40 \mathrm{~dB}$; sampling frequency is $3 \mathrm{MHz}$; peak identification time is 50 us; impact identification time is 300 us; and impact locking time is 1000 us. Before actual monitoring, the pencil lead break test is essential to be performed for determining and calibrating the test system.

\section{AE Parameters Analysis Methods}

3.1. $b$ Value. The AE peak amplitude is related to the microstructural and macrostructural cracks [28]. The $b$ value is a statistical analysis of the AE amplitude during fracture. It originates from seismology and can be used for quantitative analysis of the fracture development in concrete [29]. The $b$ value can be depicted as follows:

$$
\log _{10} N(M)=a-b\left(\frac{A_{d B}}{20}\right)
$$

where $A_{d B}$ represents the peak amplitude of $\mathrm{AE}$ events, and $N(M)$ is the number of AE events of magnitude $>=$ M. $a$ and $b$ are the fitting coefficients related to the scale of the structural crack [30]. During the fracture process, if smallscale fractures are dominant, a relatively high $b$ value is obtained, and conversely, a low $b$ value indicates the prevalence of macrocrack.

3.2. Basic Theory of Multifractal Theory. The transformation of structure in time and space can be described quantitatively and qualitatively using multifractal, which is a promising prognostic and diagnostic tool [16]. In the part, the multifractal of $\mathrm{AE}$ hits is calculated to detailed description of the occurrence and expansion of concrete failure cracks. Suppose a correlated series of signal $\left\{u_{k}, 1, \ldots, N\right\}$, the multifractal can be conducted with the following steps:
(1) Determine the profile $Y(i)[31]$ :

$$
Y(k)=\sum_{k=1}^{N}\left(u_{k}-\left\langle x_{k}\right\rangle\right), \quad k=1, \ldots \infty, N,
$$

where $\left\langle x_{k}\right\rangle$ is the average of $\left\{u_{k}\right\}$.

(2) The profile $Y(k)$ is further partitioned into $N s=$ int $(N / s)$ nonoverlapping segments with equal length $s$. The same procedure is repeated from the end of the profile for the complete use of the data. Thus, $2 \mathrm{Ns}$ segments are obtained.

(3) For 2 Ns segments, the local trend is evaluated by the least-square fitting polynomial, and the variance is calculated:

$$
F^{2}(v, s)=\frac{1}{s} \sum_{k=1}^{s}\left(Y[(v-1) s+k]-y_{v}(k)\right)^{2}, \quad v=1, \ldots, N_{s},
$$

where $y_{v}(k)$ is the polynomial regression fit for each of the 2 Ns.

(4) The $q^{\text {th }}$ order detrended (with no trend) covariance is calculated by the average over all segments:

$$
F_{q}(s)=\left\{\frac{1}{2 N_{s}} \sum_{v=1}^{2 N_{s}}[F(v, s)]^{(q / 2)}\right\}^{(1 / q)}
$$

where $q$ is an index, and $F_{q}(s)$ is the detrended covariance.

(5) The scaling characteristics of fluctuation can be determined by analyzing the log-log plots $F_{q}(s)$ versus $s$ for each value of $q[32,33]$. The generalized Hurst exponent of $h(q)$ is obtained by the slope of the linear regression of $\log F_{q}(s)$ versus $\log (s)$ [34].

According to the standard allocation function, the relationship between scale function $\tau(q)$ and Hust index $h(q)$ can be expressed as follows:

$$
\tau(q)=q h(q)-1 .
$$

The singularity strength $\alpha$ and the singularity spectrum $f$ $(\alpha)$ can be calculated with the following equations:

$$
\begin{aligned}
\alpha=\frac{\mathrm{d} \tau(q)}{\mathrm{d} q} & =h(q)+q h^{\prime}(q), \\
f(\alpha) & =q\left(a-h_{q}\right)+1 .
\end{aligned}
$$

The shape and parameters of multifractal is a way to qualitatively and quantitatively describe the complex signal. The shape of the multifractal spectrum deviates to the left side, a long tail in right called the sparse spectrum, indicating that small signals occupy a large proportion. In other words, the shape of the multifractal spectrum deviates to the right side, a long tail in left called the intensive spectrum, which indicated that large signals contribute to the dominance in the discreteness of signals [18]. Furthermore, the overall Hurst exponent $\left(\alpha_{0}\right)$ and the width of the spectrum $\left(\triangle \alpha=\alpha_{\max }-\alpha_{\min }\right)$ demonstrate the density and degree of signals, respectively. A high value of $\alpha_{0}$ can be considered 
more intensive and complex than one with the opposite characteristics [35]. Moreover, the value of $\Delta \alpha$ can be used to measure of the degree of multifractal of the signal. The asymmetry parameter $r=\left(\alpha_{\max }-\alpha_{0}\right) /\left(\alpha_{0}-\alpha_{\max }\right)$ of the spectrum shape denotes the scaling behavior of fluctuations. There are two forms of multifractal spectrum symmetry, namely, (1) "left-skewed shape," in which $r$ is less than 1, indicating large fluctuations in the data, and "right-skewed shape," where $r$ is much greater than 1 ; in this case, small fluctuations play an important role in the data [17].

3.3. The FCM Algorithm. It is well known that the characteristics of AE parameters can be used to reflect the damage mechanisms of concrete. In the section, the FCM clustering algorithm is attempted to identify damage mechanisms during concrete failure. Suppose that a set of the signal $Z=\left\{z_{1}, z_{2}, \ldots, z_{n}\right\}$. The purpose of FCM is to assign signal $Z$ to $C$ categories $(2 \leq c<n)$. Following the principle, it is also possible to determine a fuzzy membership matrix $U=\left[u_{i k}\right]_{c \times n}$ and clustering center matrix $V=\left[v_{1}, v_{2}, \ldots, v_{c}\right]^{\mathrm{T}} . u_{i k}$ satisfies $u_{i k} \in[0,1], \sum_{i=1}^{c} u_{i k}=1$, and $\sum_{k=1}^{c} u_{i k}>0 . v_{i}$ represents the clustering center, $1 \leq k \leq n$, $1 \leq i \leq c$. The objective function of the FCM clustering algorithm is given by the following relation [36].

$$
\min J(U, V)=\sum_{k=1}^{n} \sum_{i=1}^{c}\left(u_{i k}\right)^{m}\left(d_{i k}\right)^{2}
$$

where $m$ represents the weight coefficient, $m \in\left[1,+{ }^{\infty}\right)$; $J(U$, $V$ ) represents the sum of squared distances from the data samples in the $c$ categories to each cluster center. The smaller the value of $J(U, V)$, the better the clustering effect. When $J$ $(U, V)$ takes the minimum value, the clustering center and membership of the algorithm are obtained, respectively [37].

$$
\begin{gathered}
P_{i} \frac{\sum_{k=1}^{n}\left(u_{i k}\right)^{m} x_{k}}{\sum_{k=1}^{n}\left(u_{i k}\right)^{m},} \\
\left.u_{i k}=\left[\sum_{n=1}^{c} \frac{\mathrm{d}_{i k}}{\mathrm{~d}_{n k}}\right]^{(2 / m-1)}\right]^{-1} .
\end{gathered}
$$

The basic flow of FCM clustering algorithm is as follows:

(1) Among all the data samples, C data are randomly selected as initial cluster center, initialize membership matrix

(2) Calculating the distance between all data samples and $\mathrm{C}$ cluster centers, update the cluster center and membership matrix

(3) Determine whether the objective function value $J$ ( $U$, $V$ ) reaches the minimum, if it is reached, it is the iteration termination condition; otherwise, repeat step 2 .

3.4. SVM Model Principles. SVM is first applied to classification problems. Subsequently, SVM is further applied to the regression and prediction problems with the introduction of the loss function $\varepsilon$-insensitive $[38,39]$. In this section, the mathematical formulation can be briefly described below.

Suppose that a set of training datasets is given as follows: $D=\left\{\left(x_{i}, y_{i}\right), i=1,2,3, \ldots, n\right\}$, where $x_{i}$ is the value of the input, $y_{i}$ is the corresponding value of the output, and $n$ is the size of the training dataset. The optimum regression function takes the following form:

$$
f(x)=\omega \cdot \phi(x)+b,
$$

where $\phi(x)$ is the mapping function, representing the highdimensional feature space nonlinearly mapped from the input space $x . \omega$ is the weight vector, and $b$ is the bias of the hyperplane. The parameters $\omega$ and $b$ of the regression function can be estimated by minimizing the regularized risk function as follows [40].

Minimize

$$
\frac{1}{2}\|\omega\|^{2}+C \sum_{i=1}^{n}\left(\xi_{i}+\xi_{i}^{*}\right)
$$

and subject to

$$
\begin{aligned}
y_{i}-\omega \cdot \phi\left(x_{i}\right)-b & \leq \varepsilon+\xi_{i}, \\
\omega \cdot \phi\left(x_{i}\right)+b & \leq \varepsilon+\xi_{i}^{*}, \\
\xi_{i}, \xi_{i}^{*} & \geq 0, \quad i=1,2, \ldots, n,
\end{aligned}
$$

where $\xi_{i}$ and $\xi_{i}^{*}$ are the slack variables. The parameter C, a positive constant, is a prespecified SVM tolerance parameter. The data points will be penalized if the error is larger than precision parameter $\varepsilon$ [41]. $\varepsilon$ is the insensitive loss function and can be written in the following way:

$$
L_{\varepsilon}(y) \begin{cases}|f(x)-y|-\varepsilon, & |y-f(x)| \geq \varepsilon, \\ 0, & \text { otherwise. }\end{cases}
$$

The introduction of Lagrangian multipliers and maximizing can be used to solve the dual optimization problem. Equation (10) can be transformed into a dual-space optimization problem which can be solved by using Lagrange multipliers. Therefore, equation (10) can be described by the following way.

Maximize

$$
-\varepsilon \sum_{i=1}^{n}\left(\alpha_{i}+\alpha_{i}^{*}\right)-\frac{1}{2} \sum_{i, j=1}^{n}\left(\alpha_{i}-\alpha_{i}^{*}\right)\left(\alpha_{j}-\alpha_{j}^{*}\right)\left\langle x_{i}, x_{j}\right\rangle+\sum_{i=1}^{n}\left(\alpha_{i}-\alpha_{i}^{*}\right) y_{i},
$$


and subject to

$$
\left\{\sum_{i=1}^{n}\left(\alpha_{i}-\alpha_{i}^{*}\right)=0,0 \leq \alpha_{i}, \alpha_{i}^{*} \leq C, \quad i=1,2,3, \ldots, n,\right.
$$

where $\alpha_{i}, \alpha_{i}^{*}$ are the Lagrangian multipliers. Once the optimized Lagrange multipliers are determined, $\omega$ and $b$ of the regression function given by equation (9) are finally achieved as follows:

$$
\begin{aligned}
f(x) & =\frac{1}{n} \sum_{i=1}^{n}\left(y_{i}-\sum_{j=1}^{n}\left(\alpha_{j}-\alpha_{j}^{*}\right)\left\langle x_{i}, x_{j}\right\rangle\right), \\
\omega & =\sum_{i=1}^{n}\left(\alpha_{i}-\alpha_{i}^{*}\right) x_{i}, \\
b & =\frac{1}{n} \sum_{i=1}^{n}\left(y_{i}-\sum_{j=1}^{n}\left(\alpha_{j}-\alpha_{j}^{*}\right)\left\langle x_{i}, x_{j}\right\rangle\right) .
\end{aligned}
$$

and subject to

$$
\begin{aligned}
& \left\{\begin{array}{l}
\sum_{i=1}^{n}\left(\alpha_{i}-\alpha_{i}^{*}\right)=0, \\
0 \leq \alpha_{i}, \alpha_{i}^{*} \leq C, \quad i=1,2,3, \ldots, n,
\end{array}\right. \\
& f(x)=\sum_{i=1}^{n}\left[\left(\alpha_{i}-\alpha_{i}^{*}\right) K\left(x_{i}, x_{j}\right)\right]+b .
\end{aligned}
$$

Several significant kernel functions in practices problems are polynomial kernel, radial basis function kernel (RBF), and sigmoid kernel functions [24]. Among them, RBF is favorable for the capability of dealing with nonlinearity and high-dimensional computation. RBF function can be stated as follows [43]:

$$
K\left(x, x^{\prime}\right)=\exp \left(-\frac{\left\|x-x^{\prime}\right\|^{2}}{2 \sigma^{2}}\right) .
$$

\section{Results and Discussion}

4.1. Crack Development. The mechanical behavior of concrete is associated with the nonlinear propagation of internal cracks. The varying AE parameters can reflect the characteristics of the crack in real time [44]. Thus, the different stages of the deformation failure process of the concrete generally can be described by $\mathrm{AE}$ parameters with the rapid release of internal energy [2]. In the part, the deformation and compressive failure process of concretes were analyzed by the counts and the cumulative amount of counts.
It should be emphasized that only some of Lagrangian multipliers may be zero and corresponds to error of training object less than $\varepsilon$. A small number of coefficients with nonzero, called support vectors, are applied to calculate $\omega$ and $b[42]$.

The training dataset always appears as the inner product of paired samples, and the advantage of representing the learner in the dual form is that the number of adjustable parameters in the representation does not depend on the number of input attributes. The kernel function increases the capacity of the linear learner by mapping the data to a highdimensional space. It can implicitly map the nonlinear training data to the high-dimensional space without increasing the number of adjustable parameters. Therefore, the solution of the nonlinear problem has the flexibility and maneuverability by using proper kernel function to replace the inner product. The nonlinear regression function can be written as follows:

Maximize
Figure 2 shows the variation of counts and cumulative counts with axial loading. As shown in Figure 2, the damage process of the specimen can be partitioned into three stages: crack closure (stage I), stable crack propagation (stage II), and unstable crack propagation (stage III). Stage I: it is clear that the cumulative amount of counts increases nonlinearly, and a large number of counts are observed. In the initial stage of loading, some of the preexisting microdefects were gradually compacted and others were converted into microscopic cracking. It is a common phenomenon observed in concrete containing the original micropore and void. Stage II: the cumulative amount of counts is approximately linearly increasing, and there is no significant fluctuation in counts. With the increase of internal pressure-shear stress, the counts generated from the sliding of mortar aggregate interface, interconnection of large pores, and matrix cracking. Stage III: with the approaching of the peak load, the cumulative amount of counts generally shows an exponential growth and counts suddenly increased. In this stage, the generation of macrocracks of the concrete progressively increases and multiple macrocracks branching continuously penetrated and is unstable expansion.

4.2. B Value of Damage Evaluation. In the previous section, the counts and cumulative amount of counts are used to reveal a qualitative relationship between counts and the magnitude of cracks. In the part, the statistical analysis of amplitude of the $b$ value was used as an index to quantitatively identify damage. 


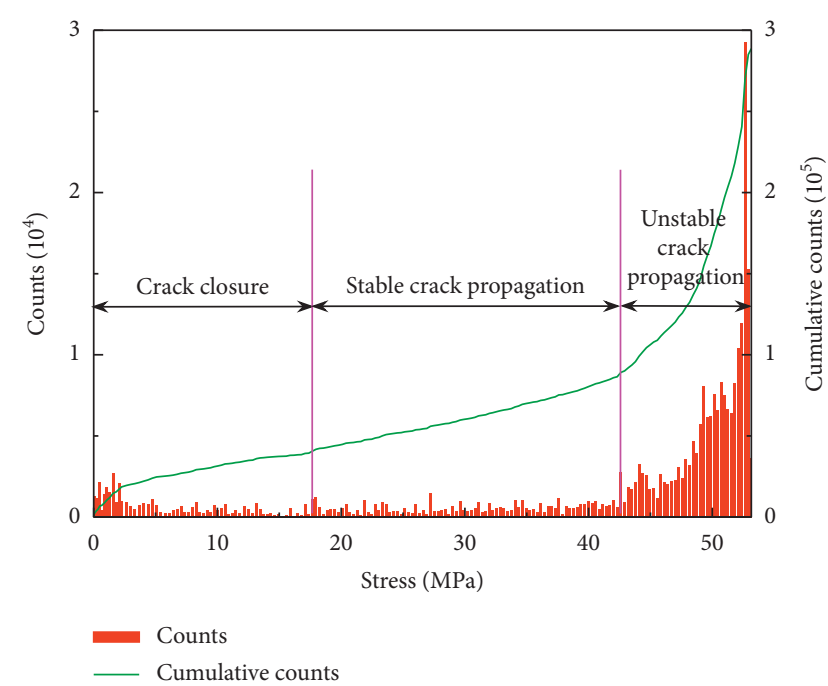

FIGURE 2: Relation between counts, cumulative counts, and stress.

The result of the evolution of the $b$ value for concrete is presented in Figure 3. It can be seen from Figure 3 that three distinct damage levels can be identified with the variation of the $b$ value. At the early stages of loading, the $b$ values ranged from 1.39 to 1.77 , which could be explained by the dominant of the microcrack. With the increase of load, the fluctuation of the $b$ value was observed in a relatively narrow range (1.44-1.74), which may be the stable macrocrack. At the near peak load, a sudden drop to 1.35 in the $b$ value was observed. This can be interpreted as a shift from local macrocrack to cohesive failure. As the stress increases to the ultimate load, the $b$ value fluctuates over a wide range due to macrocrack and microcrack.

\subsection{Multifractal Analysis of Damage Evaluation.} Although the $b$ value analysis can be used to identify microfractures and macrofractures, a major criticism of the results is limited in the analysis of fracture mode characterization due to the irregular multiple peaks [45]. Due to the self-similarity and multiscale of concrete cracking, the release of AE signals may follow the fractal characteristics in time and space distribution [15]. Therefore, the multifractal analysis of AE signals is attempted to provide a quantitative index of the complexity of the multiscale of crack.

For the purpose of qualitative and quantitative evaluation of damage in the concrete failure process, the morphology and parameters of the multifractal spectrum of $\mathrm{AE}$ hits under three stages were analyzed. The morphology of multifractal curves is shown in Figure 4, which allows us to compare the differences of the multifractal characteristic curves. From Figure 4, we can see that multifractal curves in the three stages are all asymmetrical, mainly the intensive spectrum. The results obtained from the preliminary analysis of the morphology of the multifractal spectrum revealed that the relatively large signals contributed to the dominance of the multifractal descriptive statistics. However, this relative size relationship was only in the statistical analysis of data. The more surprising correlation is with the stress level and

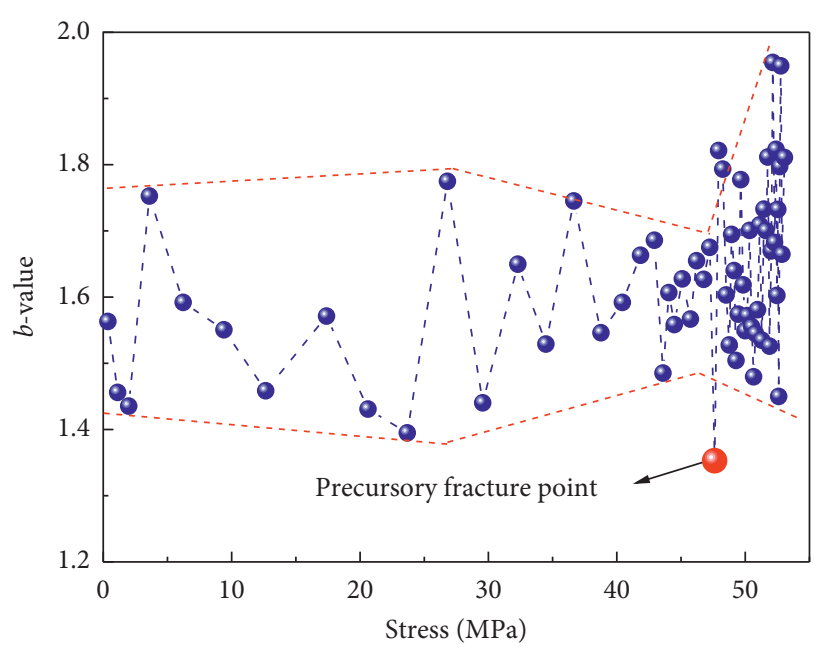

FIgURE 3: Relation between b value and stress.

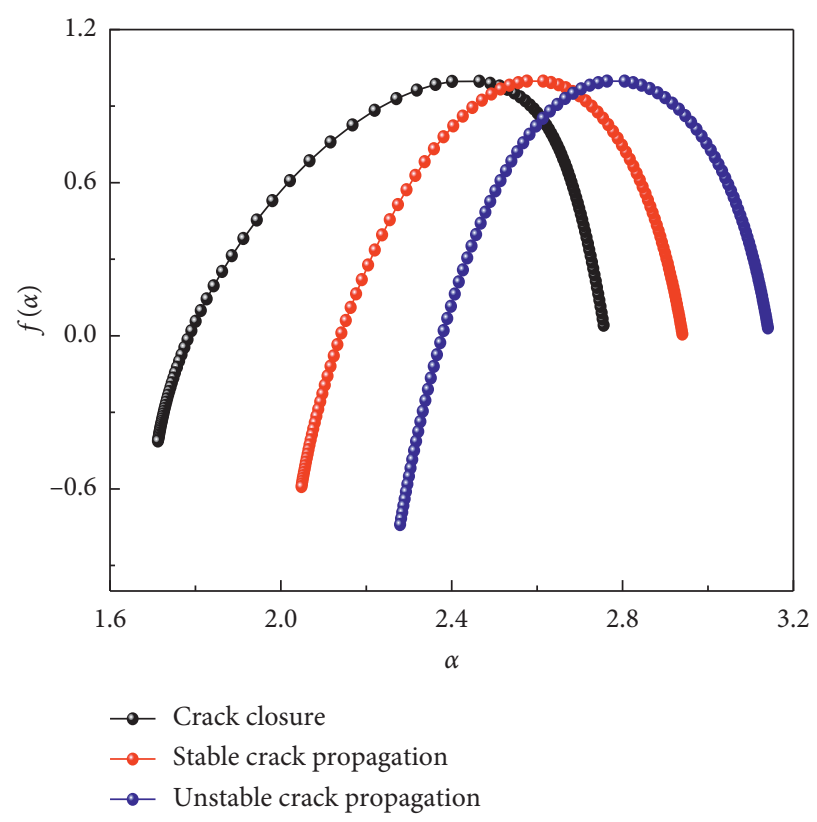

Figure 4: Multifractal spectrum at different stages.

the multifractal characteristic curves. With the stress increasing, there is a clear trend of decreasing proportion in the left part of the spectrum due to the heterogeneity of crack morphology.

In order to further explore the quantitative relationship between the multifractal parameters and damage, the variability of $\Delta \alpha, r$, and $\alpha_{0}$ was calculated and is listed in Table 2. It can be seen from the data in Table 2 that there is a clear trend of decreasing for the variability of $\Delta \alpha$ and $r$ and increasing in $\alpha_{0}$. During the whole failure process, the value of $r$ was greater than 1 , which indicated a left-skewed spectrum. The characteristics of the left-skewed spectrum revealed the small fluctuations in the AE signal according to multifractal statistics. In addition, the parameter of $r$ shows a downward trend which suggested that the fluctuation in the data is 
TABLE 2: Multifractal values for $r, \Delta \alpha$, and $\alpha_{0}$.

\begin{tabular}{lccc}
\hline & $\begin{array}{c}\text { Crack } \\
\text { closure }\end{array}$ & $\begin{array}{c}\text { Stable crack } \\
\text { propagation }\end{array}$ & Unstable crack propagation \\
\hline$r$ & 2.59 & 1.74 & 1.57 \\
$\Delta \alpha$ & 1.04 & 0.89 & 0.86 \\
$\alpha_{0}$ & 2.47 & 2.61 & 2.80 \\
\hline
\end{tabular}

increasing. Then, $\Delta \alpha$ continuous reduction from 1.04 to 0.86 indicates the degree of multifractal decrease in AE series. However, the variability of $\alpha_{0}$ is shown to be increasing. What is interesting in these data is that there is a significant positive correlation between $\alpha_{0}$ and crack density. In other words, the high value of $\alpha_{0}$ corresponded to a high cracking density.

The analysis of multifractal can provide information on the density, complexity, fractal characteristics, and anisotropy of crack. Thus, the part sets out with the aim of assessing the importance of multifractal parameter in quantitative description of damage of concrete. The curvature and symmetry of multifractal spectrum indicate that the relatively large signals occupy a large proportion. However, the findings of the current study do not support the previous analysis of $b$, which indicates that small amplitude events occur more frequently. A possible explanation for the results may be attributed to the difference in statistical parameters and methods. Compared with the variability of $\Delta \alpha, r$, and $\alpha_{0}$ in three stages, parameters vary more from stage I to stage II than they do from stage II to stage III. This discrepancy could be attributed to the cracking mechanisms. In stage I, the AE signals are mainly due to the closure of fissures and microcrack. However, macroscopic cracking is the main factor of signals in stage II and III. Table 2 shows that there has been a fall in the parameter of $r$. It is necessary here to clarify exactly what is meant by expanding macroscopic cracking.

The most striking result to emerge from the data is $\alpha_{0}$. Figure 5 provides the summary statistics for $\alpha_{0}$. As shown in Figure 5 , in the whole process of concrete failure, $\alpha_{0}$ shows the characteristics of phase change, which is similar to the trend of counts and the value $b$. At the initial loading stage, there is a clear trend of increasing in $\alpha_{0}$. When concrete was loaded from $20 \mathrm{MPa}$ to $43 \mathrm{MPa}$, the value of $\alpha_{0}$ fluctuated within a small range of 2.5. Finally, when concrete was loaded to failure strength, sample had entered into the fracture stage. The variability of $\alpha_{0}$ is consistently greater than 2.5, and as the stress increases, it first rises to the maximum and then drops to 2.5. Together, these results provide important insights into multifractal parameters to quantitatively describe in the damage.

The multifractal distribution characteristics of the $\mathrm{AE}$ activities are associated with the formation and expansion of crack in materials [20]. The dynamic multifractal variability of $\alpha_{0}$ is inevitably related to the nonlinear process of cracks. In other words, the value $\alpha_{0}$ can be used to quantify the generation and development of damage. In stage I, due to the closure of microscopic defects and internal cracks of material, the signal complexity increases, which results in the discrete and decentralized in signal distribution. Figure 5

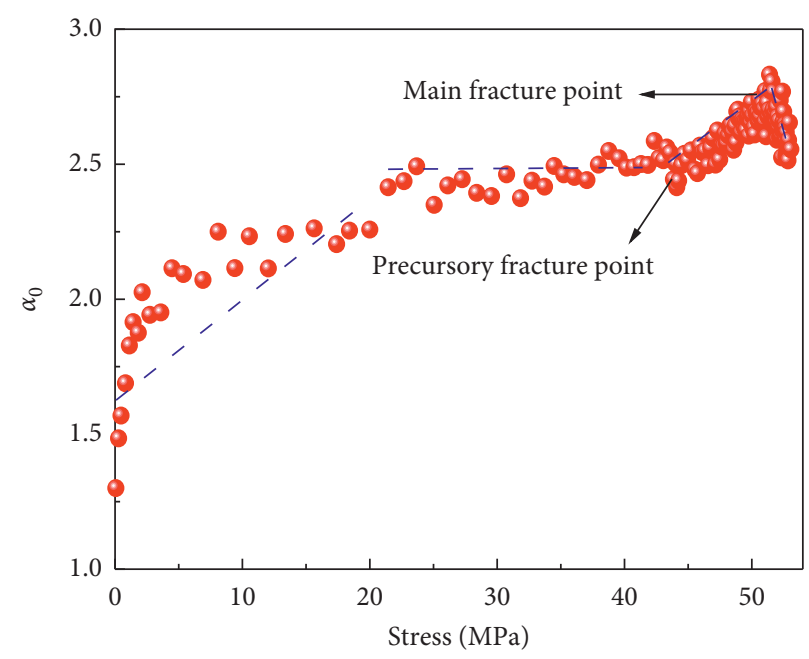

FIgURE 5: Multifractal parameter $\left(\alpha_{0}\right)$ changes with time.

shows that there has been a considerable increase in the discreteness of $\alpha_{0}$. When sample had entered into stage II, the microscopic cracking transforms into macrocrack and stable propagation, resulting in the ratio of the strength of the AE signals to remain stable. Therefore, the variations of $\alpha_{0}$ floated near 2.5. With increasing stress, the unstable expansion gradually formed the main cracking surface, which increased the crack density and complexity. Thus, $\alpha_{0}$ steadily increased. Once the main cracking surface is formed, the discrete and disordered cracks turn into the main failure surfaces. Figure 5 reveals that $\alpha_{0}$ has been a gradual drop to 2.5 until the final destruction. The present results are significant in at least major two respects. First, $\alpha_{0}$ can be used as the damage warning point if it is continuously greater than 2.5. Second, the maximum value of $\alpha_{0}$ indicates the formation of the main failure surface. This finding is of great significance for damage identification of multifractal parameters.

4.4. Identification Damage Classification Based on FCM. Due to the complexity of the damage behavior of concrete, the cluster could provide an effective and feasible method to identify the internal damage mechanisms [46]. The pertinent parameters of waveform signal are associated with damage [47]. In the part, only hits and energy are considered as input parameters of FCM to identify the type of damage mechanism of concrete in static compression tests. It should be emphasized that the three clusters are divided corresponding to three main damage mechanisms: interface cracking, matrix failure, and instability of very few aggregates near the ultimate strength.

According to the waveform characteristics of the material destruction process, the quantitative distribution of three clusters of hits and energy is shown in Figure 6. It is clearly observed in Figure 6 that the three damage mechanisms can be distinguished significantly on hits, but the difference in energy is not obvious, which is similar in [48]. The interface cracking is roughly in the range of $0-2453$ in hits, matrix cracking is in the range of 2453-5480, and 


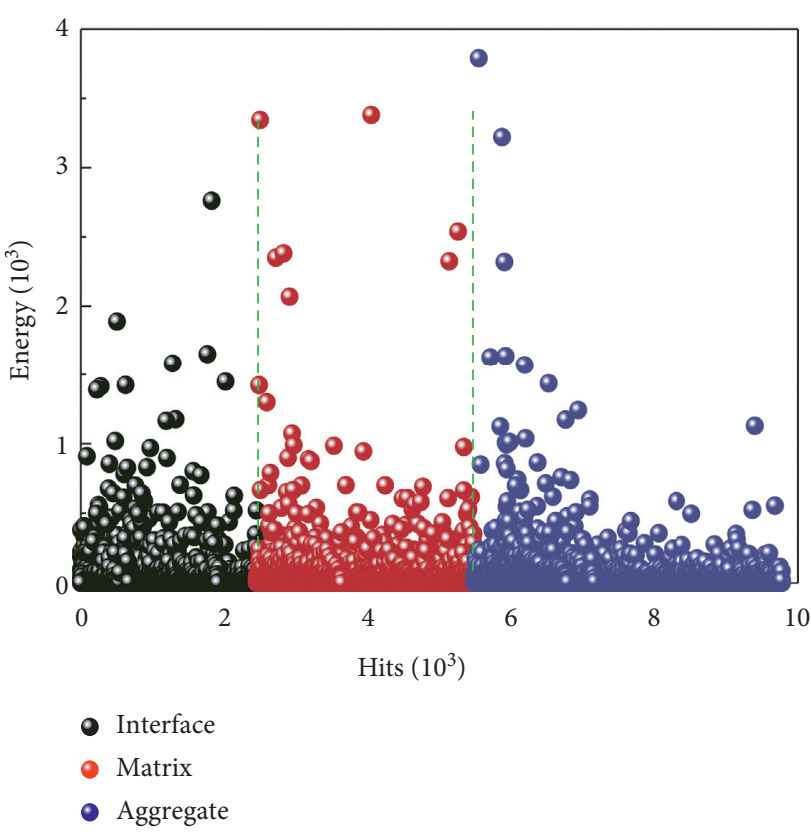

Figure 6: Distribution of energy versus hits.

aggregate damage is greater than 5480 in hits. It can be seen from the above results that the damage mechanisms can be accurately and quantitatively identified in hits and energy based on FCM.

To further explore the accuracy of mechanism identification, the cumulative hits of damage and stress are shown in Figure 7. AE activity corresponding to the interface was found during the loading process. It should be emphasized, however, that only the interface cracking signal exists in the initial damage stage. This could explain the degradation in interface properties. The intuitive results show that the matrix cracking generates as it begins from approximately $27 \mathrm{MPa}$. The slope of cumulative hits of matrix cracking is constant between $27 \mathrm{MPa}$ and $47 \mathrm{MPa}$, due to stable evolution of crack. With the continuing increase of stress until failure, the number of hits corresponding to the matrix is significantly increasing, due to the dramatic nonlinear expansion of cracks in the matrix. Moreover, the activities corresponding to aggregates appear and rapid growth.

\subsection{Development of SVM for Prediction of CS}

4.5.1. SVM Model Preparation and Preprocessing. SVM is an effective technology for estimation. Mixture components of concrete are frequently regarded as the main input parameter to predict the strength of concrete [23]. In this study, AE parameters of cumulative counts (CC), cumulative energy (CE), and cumulative hits $(\mathrm{CH})$, in the concrete compression process, are used as input parameters to predict the CS of concrete. The corresponding AE data (CC, CE, and $\mathrm{CH}$ ) in the compression process are shown in Figure 8. It can be seen from Figure 8 that $\mathrm{CC}, \mathrm{CE}$, and $\mathrm{CH}$ have different orders of magnitude. Thus, in order to implement SVM models, the database is randomly selected with 60 data from the collected data and was partitioned into two subsets: 40

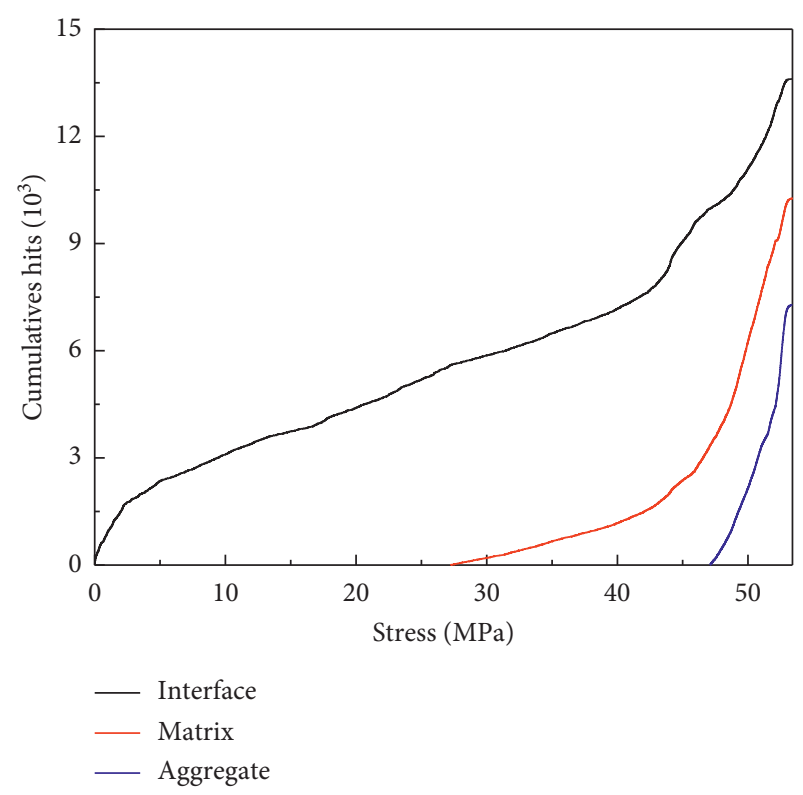

FIGURE 7: Cumulative number of hits of each cluster versus stress.

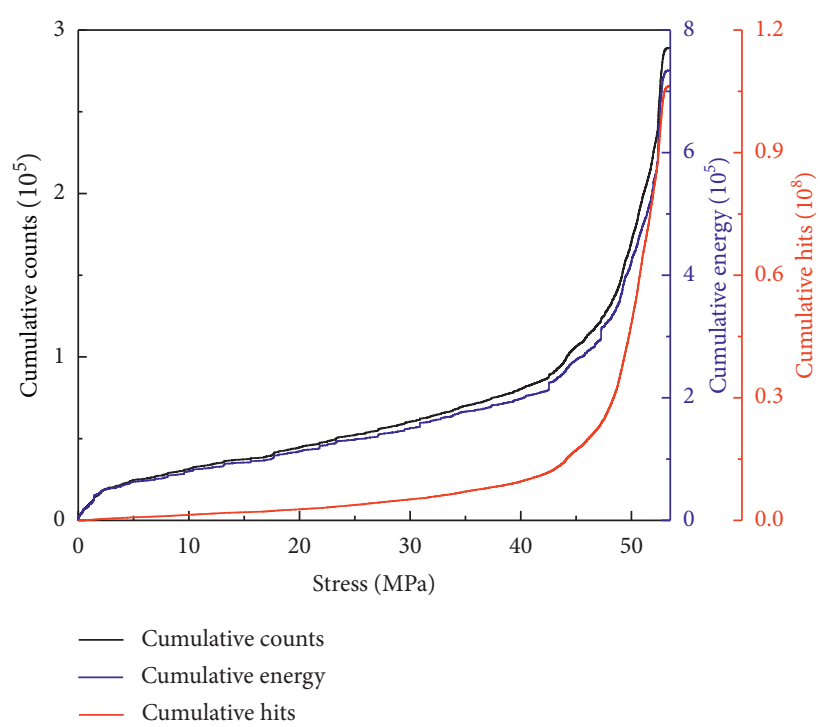

FIGURE 8: Relation between cumulative counts, cumulative energy, cumulative events, and stress.

training and 20 testing. Training and testing databases are listed in Table 3.

The training is required to develop the model of recognizing the patterns between input and output data. The testing is used for the evaluation of the performance of developed models. The speed and accuracy of prediction calculations can be increased by normalizing the input data. Hence, the input and output data are normalized before being used in the SVR model as follows [49]:

$$
x_{i}^{n}=\frac{x_{i}^{a}-x_{i}^{\min }}{x_{i}^{\max }-x_{i}^{\min }},
$$


TABLE 3: Training and testing databases.

\begin{tabular}{|c|c|c|c|c|}
\hline Training sample & $\mathrm{CC}\left(\times 10^{5}\right)$ & $\mathrm{CE}\left(\times 10^{5}\right)$ & $\mathrm{CH}\left(\times 10^{8}\right)$ & $\mathrm{CS}(\mathrm{MPa})$ \\
\hline 1 & 2.76 & 7.01 & 1.00 & 52.74 \\
\hline 2 & 2.70 & 6.85 & 0.96 & 52.64 \\
\hline 3 & 2.68 & 6.81 & 0.96 & 52.62 \\
\hline 4 & 2.65 & 6.70 & 0.95 & 52.59 \\
\hline 5 & 2.65 & 6.70 & 0.95 & 52.59 \\
\hline 6 & 2.64 & 6.68 & 0.95 & 52.59 \\
\hline 7 & 2.61 & 6.52 & 0.94 & 52.57 \\
\hline 8 & 2.53 & 6.32 & 0.91 & 52.47 \\
\hline 9 & 2.51 & 6.29 & 0.90 & 52.46 \\
\hline 10 & 2.48 & 6.21 & 0.89 & 52.44 \\
\hline 11 & 2.48 & 6.20 & 0.89 & 52.44 \\
\hline 12 & 2.38 & 5.85 & 0.87 & 52.37 \\
\hline 13 & 2.38 & 5.84 & 0.87 & 52.36 \\
\hline 14 & 2.33 & 5.68 & 0.85 & 52.24 \\
\hline 15 & 2.31 & 5.64 & 0.84 & 52.18 \\
\hline 16 & 2.31 & 5.64 & 0.84 & 52.18 \\
\hline 17 & 2.25 & 5.49 & 0.80 & 51.97 \\
\hline 18 & 2.24 & 5.46 & 0.80 & 51.96 \\
\hline 19 & 2.18 & 5.28 & 0.77 & 51.83 \\
\hline 20 & 2.12 & 5.14 & 0.73 & 51.61 \\
\hline 21 & 2.10 & 5.09 & 0.72 & 51.53 \\
\hline 22 & 2.10 & 5.07 & 0.72 & 51.52 \\
\hline 23 & 2.08 & 5.03 & 0.71 & 51.43 \\
\hline 24 & 2.04 & 4.93 & 0.69 & 51.29 \\
\hline 25 & 2.03 & 4.92 & 0.68 & 51.25 \\
\hline 26 & 2.00 & 4.85 & 0.66 & 51.13 \\
\hline 27 & 1.97 & 4.78 & 0.64 & 50.97 \\
\hline 28 & 1.84 & 4.52 & 0.56 & 50.56 \\
\hline 29 & 1.75 & 4.32 & 0.50 & 50.14 \\
\hline 30 & 1.70 & 4.21 & 0.48 & 50.00 \\
\hline 31 & 1.70 & 4.21 & 0.48 & 49.98 \\
\hline 32 & 1.68 & 4.16 & 0.47 & 49.90 \\
\hline 33 & 1.67 & 4.14 & 0.46 & 49.88 \\
\hline 34 & 1.66 & 4.11 & 0.46 & 49.83 \\
\hline 35 & 1.62 & 4.03 & 0.43 & 49.65 \\
\hline 36 & 1.62 & 4.02 & 0.43 & 49.63 \\
\hline 37 & 1.61 & 4.00 & 0.42 & 49.56 \\
\hline 38 & 1.56 & 3.90 & 0.40 & 49.35 \\
\hline 39 & 1.38 & 3.41 & 0.31 & 48.51 \\
\hline 40 & 1.36 & 3.39 & 0.30 & 48.42 \\
\hline 41 & 1.33 & 3.31 & 0.29 & 48.19 \\
\hline 42 & 1.31 & 3.27 & 0.28 & 48.01 \\
\hline 43 & 1.29 & 3.25 & 0.27 & 47.88 \\
\hline 44 & 1.29 & 3.24 & 0.26 & 47.81 \\
\hline 45 & 1.18 & 2.84 & 0.22 & 46.69 \\
\hline 46 & 1.16 & 2.82 & 0.21 & 46.55 \\
\hline 47 & 1.16 & 2.82 & 0.21 & 46.53 \\
\hline 48 & 1.14 & 2.77 & 0.20 & 46.07 \\
\hline 49 & 1.11 & 2.71 & 0.19 & 45.77 \\
\hline 50 & 1.10 & 2.70 & 0.19 & 45.75 \\
\hline 51 & 1.08 & 2.65 & 0.18 & 45.30 \\
\hline 52 & 1.08 & 2.65 & 0.18 & 45.21 \\
\hline 53 & 1.07 & 2.64 & 0.18 & 45.17 \\
\hline 54 & 1.04 & 2.57 & 0.16 & 44.62 \\
\hline 55 & 0.93 & 2.34 & 0.13 & 43.43 \\
\hline 56 & 0.92 & 2.31 & 0.13 & 43.24 \\
\hline 57 & 0.92 & 2.31 & 0.13 & 43.23 \\
\hline 58 & 0.91 & 2.29 & 0.13 & 43.13 \\
\hline 59 & 0.86 & 2.10 & 0.11 & 42.14 \\
\hline 60 & 0.81 & 2.00 & 0.10 & 40.24 \\
\hline
\end{tabular}

where $x_{i}^{a}$ and $x_{i}^{n}$ are the $i^{\text {th }}$ input vectors before and after normalization, respectively. $x_{i}^{\max }$ and $x_{i}^{\min }$ are the maximum and minimum values of the corresponding input vector.

As mentioned previously, the database was partitioned into two subsets: training and testing. The SVM model randomly selected 40 data of the total database. 80 data were trained, and the other 20 data were for testing. The $\mathrm{AE}$ parameters (CC, $\mathrm{CE}$, and $\mathrm{CH}$ ) were used as the input data of the SVM model, and the corresponding CS was used as the output data.

Parameters $C, \varepsilon$, and $\sigma$ are the key parameters of the SVM model, which can affect the calculation speed and accuracy of the model. In the present study, optimal values of parameters $(C, \varepsilon$, and $\sigma)$ are selected by grid search. The training and testing process of one specimen is shown in Figure 9.

The reliability of the SVM model is also evaluated via the following statistical parameters: RMSE, MAPE, and the correlation coefficient of between predicted and actual results (R2). These statistical parameters can reflect the accuracy of predicting results. The calculation formulas of RMSE, MAPE, and R2 have been given in literature $[49,50]$.

4.5.2. Analysis of the SVM Prediction Performance. To further expand the application of AE for predicting compressive of concrete. SVM is developed to estimate the concrete CS based on AE parameters as inputs. Figure 10 illustrates the typical characteristics of the relationship between the prediction results and the actual concrete CS. We obtained the correlation coefficients $R^{2}$ approximately equal to 1 . It is indicated that CS obtained from the proposed SVM model is in pleasurable agreement with the experimental value.

Figure 11 compares MAPE and RMSE values in ANN and SVM. It can be seen from Figure 11 that SVM obtains the superior value of MAPE and RMSE compared to ANN. ANN and SVM have the same application to regression problem, whereas SVM has a firmly theoretical and mathematical foundation compared to ANN, which reply on the designer's empirical knowledge and prior knowledge. SVM implements the SRM principle which aims at minimizing a bound on the generalization error of a model rather than minimizing the error on the training data only. This difference equips SVM with a greater ability to avoid overtraining and has better generalization capability than the ANN model [21]. In addition, the performance of the ANN model depends too heavily on the sample size in the process of model training. In most cases, the sample size is limited. However, SVM can achieve higher generalization ability with a limited number of samples in comparison to ANN. Thus, it is a broad indication that SVM can be regarded as a useful modeling tool for concrete.

4.5.3. Performance Comparison of AE Parameters and Others. In order to highlight the superiority of AE parameters as model input, the results of this study were 


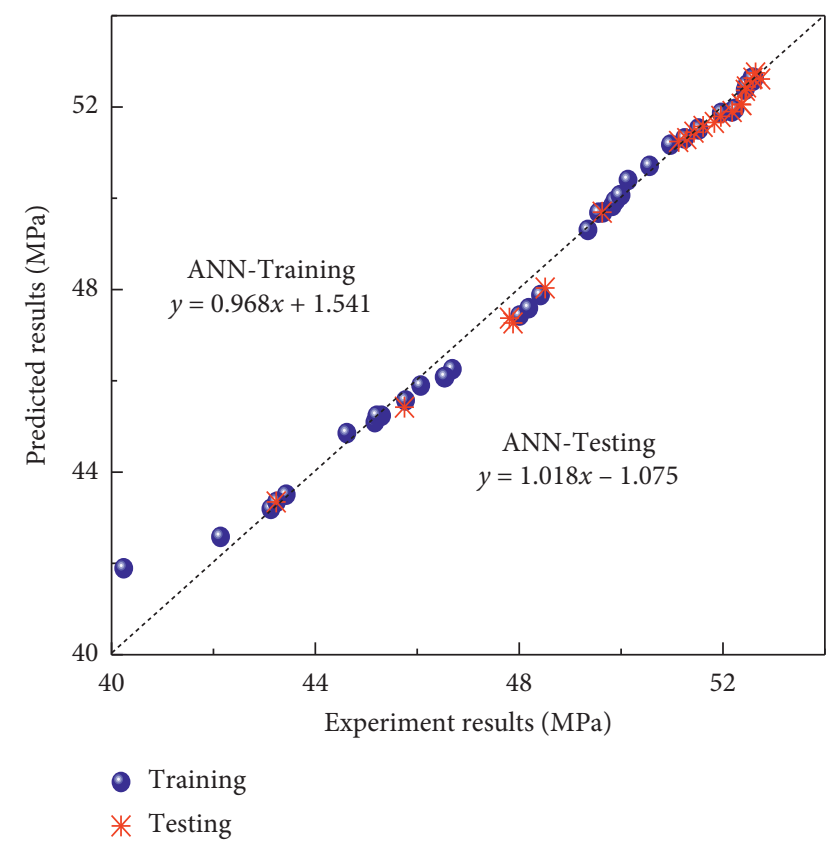

FIGURE 9: Comparison of predicted and experimental results with training and testing.

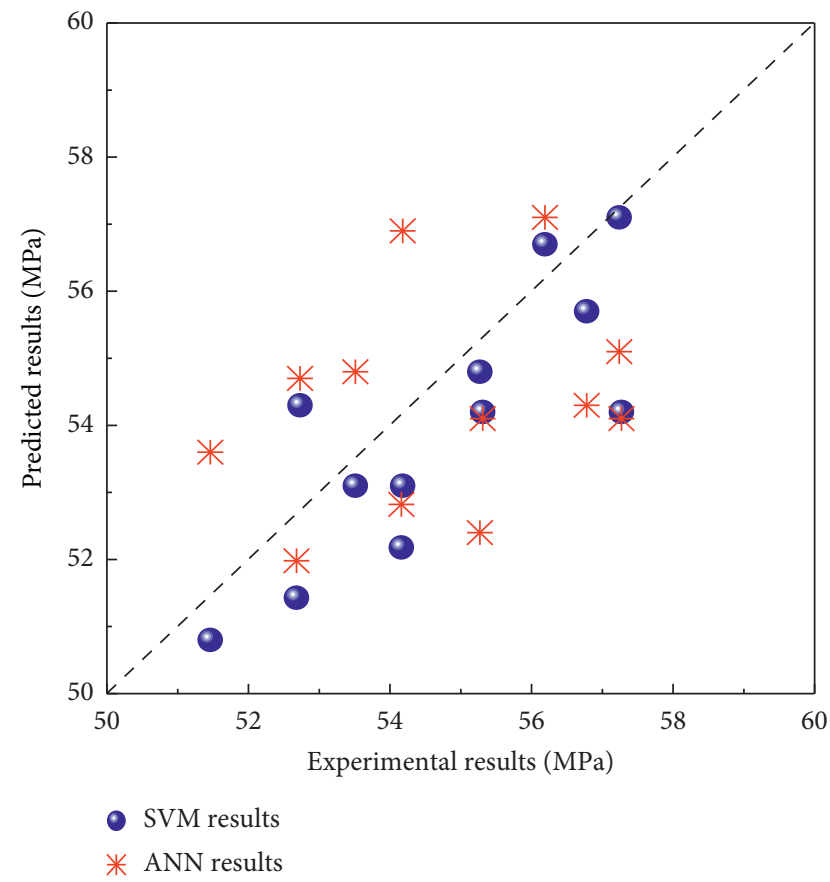

FIGURE 10: Experimental versus predicted CS.

compared with the literature $[23,51,52]$. The input parameters of Shih, Aiyer, and Cheng are ultrasonic and the composition of 6 and 8 mixtures [23, 51, 52]. The quantitative description of the accuracy of prediction results can be characterized by MAPE and RMSE.

Figure 12 shows the tendencies of MAPE and RMSE values with $\mathrm{AE}$ parameters and other three different input data. It can be seen from Figure 12 that the values of RMSE

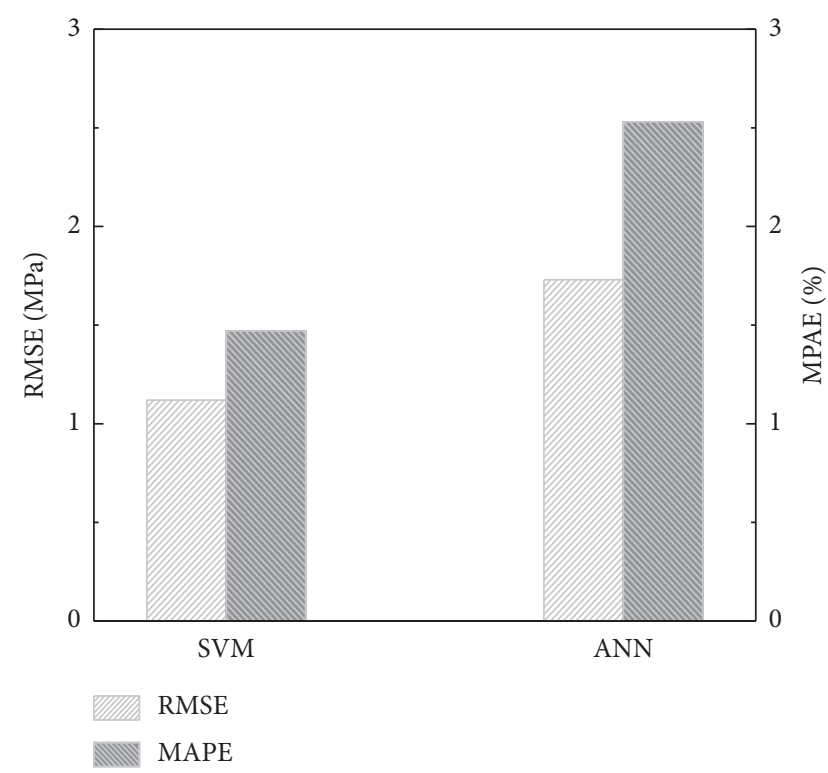

FIGURE 11: Comparison between SVM and ANN

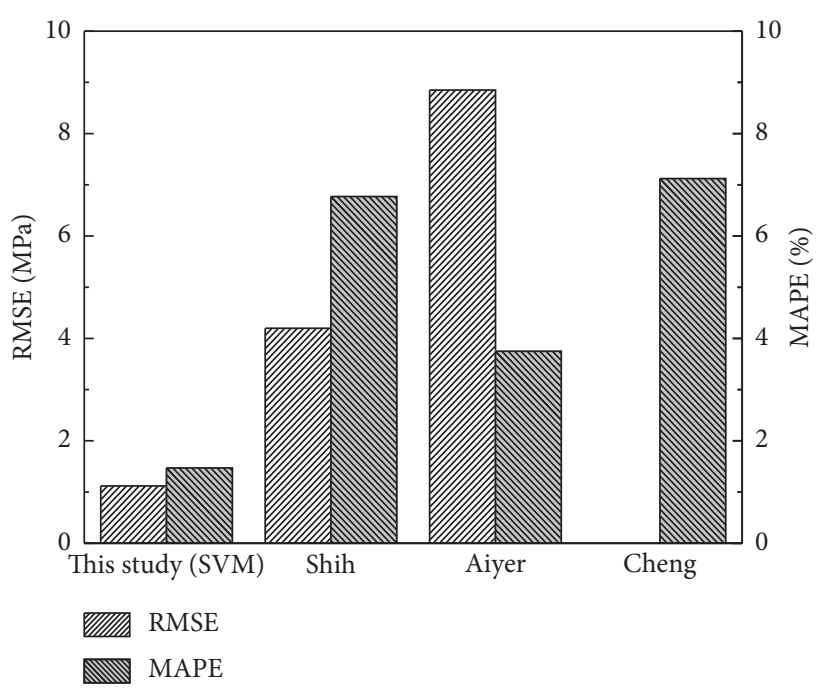

FIgURE 12: Comparison between AE parameters and other parameters in terms of RMSE and MAPE.

and MAPE of SVM using AE parameters as input were $0.74 \mathrm{MPa}$ and $1.00 \%$, respectively. Nevertheless, the values of RMSE reached separately 4.21 MPa and 8.85 MPa in [51, 52], and the values of MAPE reached separately $6.77 \%, 3.75 \%$, and $6.77 \%$ in $[23,51,52]$. It can be concluded that $\mathrm{AE}$ parameters as input data give the excellent value. In this context, AE parameters and corresponding loads in the concrete compression process are adopted as input and output of the SVM model, which can effectively reduce the number of concrete samples while meeting enough training and test samples, compared with other three input parameters. Therefore, it means that AE parameters, as the input of the model, can be used to predict CS.

Table 4 lists two differences between this study and the others, input and sample size. As can be seen from Table 3, 
TABLE 4: . Comparison between the study and others in term of input and sample number.

\begin{tabular}{lcccc}
\hline & The study & Shih [23] & Aiyer [51] & Cheng [52] \\
\hline SVM input & AE parameters & Hammer test, ultrasonic pulse velocity & 6 mixtures & 8 mixtures \\
\hline Sample size & 16 & 95 & 80 & \\
\hline
\end{tabular}

AE parameters are taken as the input of SVM, and the number of specimens is significantly reduced. In this study, only 16 samples are needed, which is far less than the number of samples in $[23,51,52]$. This is related to the sample selection of training and testing database of SVM. The characteristic of SVM is that it requires a sea of samples for training. In this study, 60 data were selected as inputs and extracted from AE data in the process of compression. Of course, more data could be extracted. One of the main advantages of $\mathrm{AE}$ parameters is to reduce the number of samples as much as possible with guaranteed accuracy.

\section{Conclusion}

This project was undertaken to establish multifractal damage analysis of AE series and propose a SVM model to predict the CS of concrete by AE parameters. Meanwhile, FCM is applied for the identification of mechanisms in concrete. Based on the investigation, the following conclusions can be drawn.

The analysis of multifractal undertaken here has extended our knowledge of the statistical methods of AE series. The morphology of the multifractal spectrum of AE parameters makes several noteworthy contributions to proportional relation of signal size with the damage level. This work contributes to quantitatively distinguish the differences of damage levels. The decrease of parameters $\Delta \alpha$ and $r$ suggests that the degree of multifractal decreases and the fluctuation increases in signals. The increase of parameter $\alpha_{0}$ is positively correlated with the density of multiple cracking. $\alpha_{0}>2.5$ indicates unstable cracks have been developed for specimen. In addition, an implication of this is the possibility that the maximum value of $\alpha_{0}$ is a fair quantitative indicator of the primary failure surface. Meanwhile, FCM has been successful in discriminating the damage mechanisms.

SVM is a powerful computational approach for analyzing the complex relationship between AE parameters and predicting concrete $\mathrm{CS}$. It is found that $\mathrm{AE}$ parameters as input of SVM have the higher precision than others by comparing MSE and MAPE. Moreover, AE parameters as inputs can greatly reduce the number of samples. In terms of forecasting models, it should be emphasized that the prediction performance of SVM is very advantageous compared with ANN. Therefore, SVM could be utilized to predict the actual failure load of concrete and may be further applied to strength prediction for civil engineers and concrete construction fields.

\section{Data Availability}

The dataset used to support the findings of this study has been deposited in the repository of OSFHOME https://osf. io/s7g3h/?view_only=0c9bacdab2c44bbdb3879c901cc0787c and is available from the corresponding author upon request.

\section{Conflicts of Interest}

The authors declare that they have no conflicts of interest.

\section{Acknowledgments}

This research was financially supported by the National Natural Science Foundation of China (51178069, 52078093, and 51974145), LiaoNing Revitalization Talents Program (XLYC1905015), and the Fundamental Research Funds Program for the Central University (FRF-BD-19-004A).

\section{References}

[1] K. V. Tittelboom, N. D. Belie, and F. Lehmann, "Acoustic emission analysis for the quantification of autonomous crack healing in concrete," Constrction and Building Materials, vol. 28, no. 1, pp. 333-341, 2012.

[2] W.-C. Choi and H.-D. Yun, "Acoustic emission activity of CFRP-strengthened reinforced concrete beams after freezethaw cycling," Cold Regions Science and Technology, vol. 110, pp. 47-58, 2015.

[3] H.-D. Yun, W.-C. Choi, and S.-Y. Seo, "Acoustic emission activities and damage evaluation of reinforced concrete beams strengthened with CFRP sheets," NDT \& E International, vol. 43, no. 7, pp. 615-628, 2010.

[4] M. Abdelrahman, M. K. Elbatanouny, and P. H. Ziehl, "Acoustic emission based damage assessment method for prestressed concrete structures: modified index of damage," Engineering Structures, vol. 60, pp. 258-264, 2014.

[5] T. Sasikumar, S. Rajendraboopathy, K. M. Usha, and E. S. Vasudev, "Artificial neural network prediction of ultimate strength of unidirectional T-300/914 tensile specimens using acoustic emission response," Journal of Nondestructive Evaluation, vol. 27, no. 4, pp. 127-133, 2008.

[6] N. Ativitavas, T. Pothisiri, and T. J. Fowler, "Identification of fiber-reinforced plastic failure mechanisms from acoustic emission data using neural networks," Journal of Composite Materials, vol. 40, no. 3, pp. 193-226, 2005.

[7] T. Chelladurai, R. Krishnamurthy, and A. R. Acharya, "An approach for the integrity assessment of M250 maraging steel pressurized systems," Journal of Acoustic Emission, vol. 8, no. $1-2$, pp. $588-591,1989$.

[8] S. Pedro, L. T. Aitor, and H. P. C. Sergio, Creep Behaviour in Cracked Sections of Fibre Reinforced Concrete, Springer, Berlin, Germany, 2017.

[9] Mizutani and Yoshihiro, Practical Acoustic Emission Testing, Springer, Berlin, Germany, 2016.

[10] N. Godin, P. Reynau, and G. Fantozzi, Acoustic Emission and Durability of Composite Materials, Springer, Berlin, Germany, 2018.

[11] D. Kocáb, L. Topolář, B. Kucharczyková, and R. Halamová, "The analysis of acoustic emission signals detected during the 
loading of cement-based materials," Engineering Failure Analysis, vol. 99, pp. 18-25, 2019.

[12] D. G. Aggelis, "Classification of cracking mode in concrete by acoustic emission parameters," Mechanics Research Communications, vol. 38, no. 3, pp. 153-157, 2011.

[13] D. G. Aggelis, A. C. Mpalaskas, and T. E. Matikas, "Acoustic signature of different fracture modes in marble and cementitious materials under flexural load," Mechanics Research Communications, vol. 47, pp. 39-43, 2013.

[14] G. Ma and Q. Du, "Structural health evaluation of the prestressed concrete using advanced acoustic emission (AE) parameters," Constrction and Building Materials, vol. 250, pp. 1-16, 2020.

[15] A. Carpinteri, G. Lacidogna, F. Accornero, A. C. Mpalaskas, T. E. Matikas, and D. G. Aggelis, "Influence of damage in the acoustic emission parameters," Cement and Concrete Composites, vol. 44, no. 44, pp. 9-16, 2013.

[16] E. A. F. Ihlen, "Introduction to multifractal detrended fluctuation analysis in matlab," Frontier and Physiol, vol. 3, no. 141, pp. 1-18, 2012.

[17] O. Sijilmassi, J. L. Alonso, and A. D. R. Sevilla, "Multifractal analysis of embryonic eye structures from female mice with dietary folic acid deficiency. Part I: fractal dimension, lacunarity, divergence, and multifractal spectrum," Chaos Solitons Fractals, vol. 138, pp. 1-13, 2020.

[18] B. Kong, E. Wang, Z. Li, X. Wang, L. Chen, and X. Kong, "Nonlinear characteristics of acoustic emissions during the deformation and fracture of sandstone subjected to thermal treatment," International Journal of Rock Mechanics and Mining Sciences, vol. 90, pp. 43-52, 2016.

[19] M. Pal and S. Deswal, "Support vector regression based shear strength modelling of deep beams," Computers \& Structctures, vol. 89, no. 13-14, pp. 1430-1439, 2011.

[20] A. Kooshkaki and H. Eskandari-Naddaf, "Effect of porosity on predicting compressive and flexural strength of cement mortar containing micro and nano-silica by multi-objective ANN modeling," Construction and Building Materials, vol. 212, pp. 176-191, 2019.

[21] P. Yuvaraj, A. Ramachandra Murthy, N. R. Iyer, S. K. Sekar, and P. Samui, "Support vector regression based models to predict fracture characteristics of high strength and ultra high strength concrete beams," Engineering Fracture Mechanics, vol. 98, pp. 29-43, 2013.

[22] J.-J. Lee, D.-K. Kim, S.-K. Chang, and J.-H. Lee, “Application of support vector regression for the prediction of concrete strength," Computers and Concrete, vol. 4, no. 4, pp. 299-316, 2007.

[23] Y.-F. Lee, Y.-R. Wang, K.-L. Lin, and C.-W. Chen, "Improving non-destructive concrete strength tests using support vector machines," Materials, vol. 8, no. 10, pp. 7169-7178, 2015.

[24] A. Nazari and J. G. Sanjayan, "Modelling of compressive strength of geopolymer paste, mortar and concrete by optimized support vector machine," Ceramics International, vol. 41, no. 9, pp. 12164-12177, 2015.

[25] A. M. Abd and S. M. Abd, "Modelling the strength of lightweight foamed concrete using support vector machine (SVM)," Case Studies in Construction Materials, vol. 6, pp. 8-15, 2017.

[26] T. Sasikumar, S. Rajendraboopathy, K. M. Usha, and E. S. Vasudev, "Failure strength prediction of unidirectional tensile coupons using acoustic emission peak amplitude and energy parameter with artificial neural networks," Composites Science and Technology, vol. 69, no. 7-8, pp. 1151-1155, 2009.
[27] JTG E30-2005, Test Methods of Cement and Concrete for Highway Engineering, Chinese Standards Institute, Beijing, China, 2005.

[28] G. Ma and H. Li, "Acoustic emission monitoring and damage assessment of FRP-strengthened reinforced concrete columns under cyclic loading," Construction and Building Materials, vol. 144, pp. 86-98, 2017.

[29] R. Vidya Sagar and M. V. M. S. Rao, "An experimental study on loading rate effect on acoustic emission based b-values related to reinforced concrete fracture," Constrction and Building Materials, vol. 70, pp. 60-472, 2014.

[30] R. V. Sagar, B. K. R. Prasad, and S. S. Kumar, "An experimental study on cracking evolution in concrete and cement mortar by the b-value analysis of acoustic emission technique," Cement and Concrete Research, vol. 42, no. 8, pp. 1094-1104, 2012.

[31] T. Takaishi, "Statistical properties and multifractality of Bitcoin," Physica A, vol. 506, pp. 1-24, 2017.

[32] M. Walid, H. Atef, and S. Yoon, "Modelling multifractality and efficiency of GCC stock markets using the MF-DFA approach: a comparative analysis of global, regional and Islamic markets," Physica A, vol. 503, pp. 1107-1116, 2018.

[33] N. Ye, C. Han, and Y. Wang, "The multifractal properties of Euro and Pound exchange rates and comparisons," Physica A, vol. 509, pp. 578-587, 2018.

[34] L. H. Fernandes, F. H. A. Araújo, and I. E. M. Silva, “The (in) efficiency of NYMEX energy futures: a multifractal analysis," Physica A, vol. 556, pp. 1-10, 2020.

[35] P. Baranowski, M. Gos, J. Krzyszczak, K. Siwek, A. Kieliszek, and P. Tkaczyk, "Multifractality of meteorological time series for Poland on the base of MERRA-2 data," Chaos, Solitons \& Fractals, vol. 127, pp. 318-333, 2019.

[36] J. Tang, S. Yu, F. Liu, X. Chen, and H. Huang, “A hierarchical prediction model for lane-changes based on combination of fuzzy C-means and adaptive neural network," Expert Systems with Applications, vol. 130, pp. 265-275, 2019.

[37] L. Liang-Qun, W. Xie, and Z. Liu, "A novel quadrature particle filtering based on fuzzy c-means clustering," Knowledge Based Systems, vol. 106, pp. 105-115, 2016.

[38] J. Tinoco, A. Gomes Correia, and P. Cortez, "Support vector machines applied to uniaxial compressive strength prediction of jet grouting columns," Computers and Geotechnics, vol. 55, pp. 132-140, 2014.

[39] M. Pal and S. Deswal, "Support vector regression based shear strength modelling of deep beams," Computers \& Structures, vol. 89, no. 13-14, pp. 1430-1439, 2011.

[40] K. Yan, H. Xu, G. Shen, and P. Liu, "Prediction of splitting tensile strength from cylinder compressive strength of concrete by support vector machine," Advances in Materials Science and Engineering, vol. 2013, pp. 1-13, 2013.

[41] J. Liu, K. Yan, X. Zhao, and Y. Hu, "Prediction of autogenous shrinkage of concretes by support vector machine," International Journal of Pavement Research and Technology, vol. 9, no. 3, pp. 169-177, 2016.

[42] R. A. Mozumder, B. Roy, and A. I. Laskar, "Support vector regression approach to predict the strength of FRP confined concrete," Arabian Journal for Science and Engineering, vol. 42, no. 3, pp. 1129-1146, 2017.

[43] K. Yan and C. Shi, "Prediction of elastic modulus of normal and high strength concrete by support vector machine," Construction and Building Materials, vol. 24, no. 8, pp. 1479-1485, 2010.

[44] J. Geng, Q. Sun, Y. Zhang, L. Cao, and W. Zhang, "Studying the dynamic damage failure of concrete based on acoustic 
emission," Construction and Building Materials, vol. 149, pp. 9-16, 2017.

[45] S. Stavroyiannis, V. Babalos, S. Bekiros, S. Lahmiri, and G. S. Uddin, "The high frequency multifractal properties of Bitcoin," Physica A: Statistical Mechanics and Its Applications, vol. 520, pp. 62-71, 2019.

[46] M. Lahmiri, A. E. Mahi, and Z. Jendli, "Static and fatigue characterization of flax fiber reinforced thermoplastic composites by acoustic emission," Applied Acoustics, vol. 147, pp. 100-110, 2018.

[47] Y. Ech-Choudany, M. Assarar, D. Scida, F. Morain-Nicolier, and B. Bellach, "Unsupervised clustering for building a learning database of acoustic emission signals to identify damage mechanisms in unidirectional laminates," Applied Acoustics, vol. 123, pp. 123-132, 2017.

[48] Y. Farnam, M. R. Geiker, D. Bentz, and J. Weiss, "Acoustic emission waveform characterization of crack origin and mode in fractured and ASR damaged concrete," Cement and Concrete Composites, vol. 60, pp. 135-145, 2015.

[49] A. R. Suleiman and M. Nehdi, "Modeling self-healing of concrete using hybrid genetic algorithm-artificial neural network," Materials, vol. 10, pp. 1-15, 2017.

[50] N. Ceryan, U. Okkan, P. Samui, and S. Ceryan, "Modeling of tensile strength of rocks materials based on support vector machines approaches," International Journal for Numerical and Analytical Methods in Geomechanics, vol. 37, no. 16, pp. 2655-2670, 2012.

[51] B. G. Aiyer, D. Kim, N. Karingattikkal, P. Samui, and P. R. Rao, "Prediction of compressive strength of selfcompacting concrete using least square support vector machine and relevance vector machine," KSCE Journal of Civil Engineering, vol. 18, no. 6, pp. 1753-1758, 2014.

[52] M.-Y. Cheng, J.-S. Chou, A. F. V. Roy, and Y.-W. Wu, "Highperformance concrete compressive strength prediction using time-weighted evolutionary fuzzy support vector machines inference model," Automation in Construction, vol. 28, pp. 106-115, 2012. 